\title{
Three cases of endoscopic resection for synchronous early colon cancers after self-expandable metallic stent placement for obstructive colon cancer
}

Authors

Institutions
Rintaro Moroi ${ }^{1,}$ *, Katsuya Endo ${ }^{2,}$ *, Ryo Ichikawa ${ }^{1}$, So Takahashi ${ }^{1}$, Takeharu Shiroki ${ }^{1}$, Hirohiko Shinkai ${ }^{1}$, Fumitake Ishiyama', Shoichi Kayaba

${ }^{1}$ Department of Gastroenterology, Iwate Prefectural Isawa Hospital, Oshu, Japan

2 Division of Gastroenterology, Department of Internal Medicine, Tohoku University Graduate School of Medicine, Sendai, Japan submitted

18. February 2016 accepted after revision 5. July 2016

\section{Bibliography}

DOI http://dx.doi.org/

10.1055/s-0042-113874

Published online: 31.8.2016

Endoscopy International Open 2016; 04: E970-E973

(C) Georg Thieme Verlag KG

Stuttgart · New York

E-ISSN 2196-9736

Corresponding author

\section{Rintaro Moroi, MD, PhD}

Department of

Gastroenterology

61 Ryugababa, Mizusawa-Ku

Oshu, Iwate, 023-0865

Japan

Fax: +81-197-24-8194

rinta@med.tohoku.ac.jp
Iwate Prefectural Isawa Hospital

Background and study aims: The feasibility of endoscopic resection for synchronous early colon cancer after placement of self-expandable metallic stents (SEMS) for malignant colorectal obstruction is unknown. Herein we evaluated 3 cases of endoscopic resection for synchronous early colorectal cancers after SEMS placement. Patient 1 was an 82-year-old man with obstructive sigmoid colon cancer. We curatively treated the synchronous descending colon cancer with endoscopic submucosal dissection (ESD) and the rectal cancer with endoscopic mucosal resection (EMR) after SEMS placement. This is the first reported case of a successful ESD for synchronous early colon cancer via the use of a colonic stent. Patient 2 was an 81-year-old man with obstructive ascend-

\section{Introduction}

$\nabla$

Self-expandable metallic stents (SEMS) are now widely used to treat malignant colonic obstruction [1-3]. In cases involving the bridge to surgery strategy, it is ideal to investigate the entire colon before surgery because the proportion of synchronous cancers in cases of obstructive colorectal cancer is $4 \%$ to $6 \%[4,5]$.

Several studies have reported the effectiveness and safety of preoperative complete colonoscopy (defined as a total colonoscopy via the use of stents) after SEMS placement [5 -7]. Preoperative complete colonoscopy has a great advantage in that, if a synchronous colorectal cancer is found, the surgical strategy can be altered to avoid multiple-stage operations. Although complete colonoscopy after SEMS placement has been recognized to be feasible and safe, the feasibility of endoscopic resection for synchronous early colon cancer (defined as carcinoma limited to the mucosa or submucosa) after SEMS placement is still

\section{License terms}

\section{()(1) $\ominus \circledast$}

* These authors contributed equally. ing colon cancer. We resected the synchronous transverse colon cancer via ESD. Histologic findings indicated that the carcinoma cells had invaded the submucosal layer. Therefore, we immediately performed expanded right-hemicolectomy. Patient 3 was an 81-year-old man with obstructive sigmoid colon cancer. We curatively treated the synchronous transverse colon cancer with EMR after SEMS placement. There were no complications associated with the endoscopic treatments in any of the cases. Our results indicate that preoperative endoscopic resection combined with the ESD technique for synchronous colorectal cancer after SEMS placement could be effective as a surgical strategy for patients with malignant colorectal obstruction.

unknown. If a synchronous early colorectal cancer is resected preoperatively using the endoscopic technique, we can evaluate its pathologic findings precisely and assess the possibility of curatively treating the lesion.

\section{Case Reports \\ V}

\section{Patient 1}

An 82-year-old man underwent SEMS for obstructive sigmoid colon cancer. After successful placement of the SEMS, we performed a scheduled second total colonoscopy and detected 3 synchronous early cancers in the ascending and descending colons and the rectum, respectively ( $\bullet$ Fig. 1). For the ascending colon cancer, which was a protruding 30-mm lesion (Type 0-Is [8]), we attempted endoscopic submucosal dissection (ESD); however, we failed to complete the procedure because of severe fibrosis in the submucosal layer. We concluded that right hemicolectomy was needed to treat the ascending colon cancer. The descending colon cancer was a flat elevated 30-mm lesion (Type 0-IIa) and the rectal cancer 


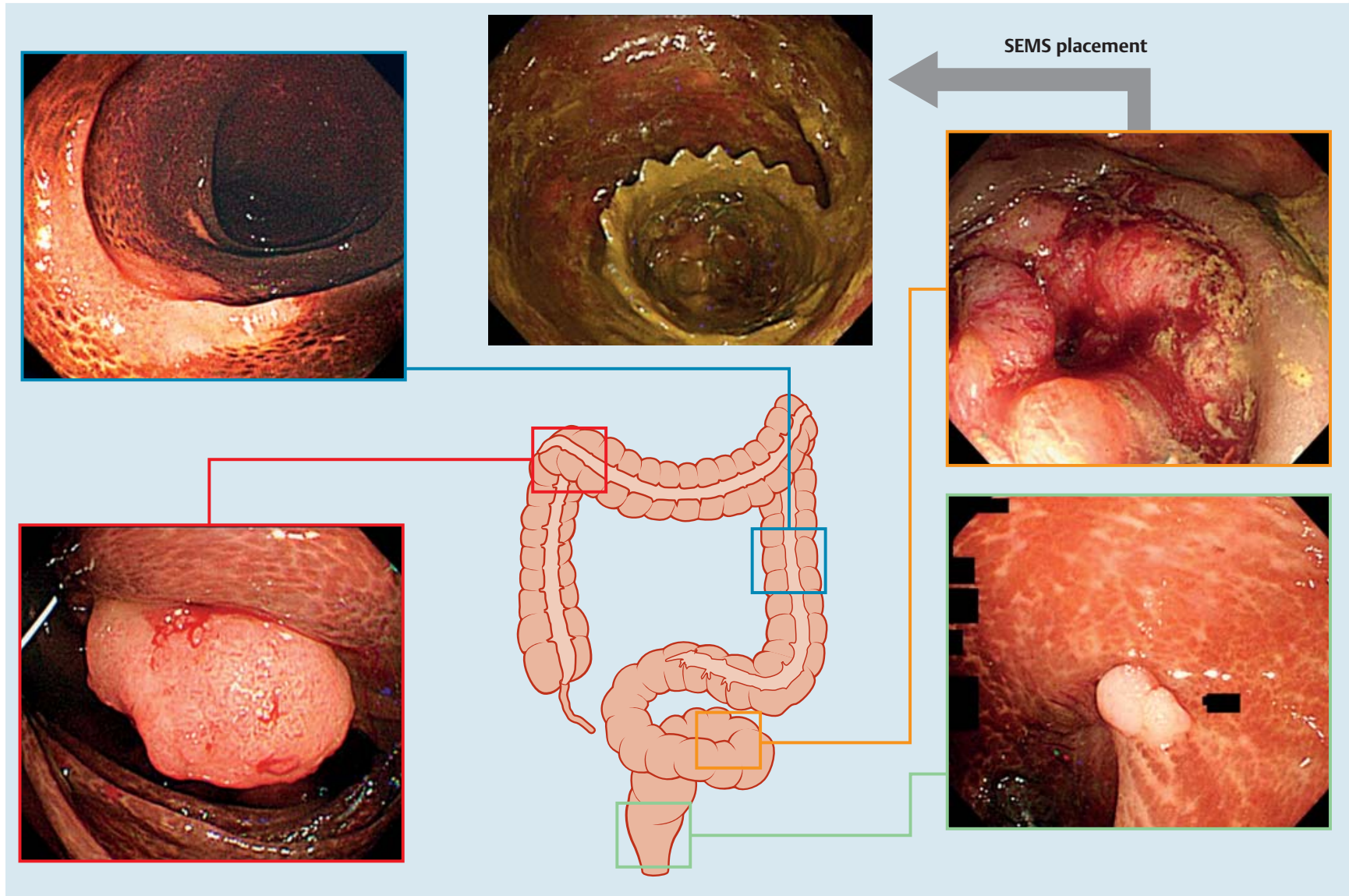

Fig. 1 SEMS placement in the sigmoid colon (orange square). In this case, melanosis coli was found in the colon. We could not accomplish ESD in the ascending colon cancer because of severe fibrosis in the submucosal layer. However, we curatively resected the descending colon cancer with ESD (blue square) and the rectal cancer with EMR (green square).

was a protruding 10-mm lesion (Type 0-Is), for which we successfully performed ESD and endoscopic mucosal resection (EMR), respectively.

The histologic findings were that both lesions were intramucosal carcinoma (pTis) and the margins were free of carcinoma cells. Therefore, we concluded that the descending colon cancer and the rectal cancer could only be curatively treated via endoscopic resection. Our findings indicated the need to perform right hemicolectomy and sigmoidectomy (pT3, pN1, cM0). Histologic findings for the ascending colon revealed that the lesion was intramucosal carcinoma (pTis) with severe fibrosis in the submucosal layer.

\section{Patient 2}

An 81-year-old man underwent SEMS for obstructive ascending colon cancer. After successful placement of the SEMS, we performed a scheduled second total colonoscopy and detected a synchronous early cancer in the transverse colon ( $\bullet$ Fig. 2 ). This mass was undetectable during stent placement because neither colonic preparation with polyethylene glycol nor precise screening for synchronous lesions was possible owing to the emergent obstruction.

For the transverse colon cancer, a flat elevated lesion with a slight depression and a diameter of $15 \mathrm{~mm}$ (Type 0-IIa + IIc), we successfully performed ESD. The histologic findings revealed that the lesion included well-differentiated adenocarcinoma cells that had invaded the submucosal layer (pT1b, SM $2200 \mu \mathrm{m}$ ). Although the margins were free of carcinoma cells, we concluded that sur- gery was necessary. We performed an expanded right hemicolectomy (pT4, pN2, pM1[H1]), including the ESD scar. No cancer cells were detected in the ESD scar.

\section{Patient 3}

An 81-year-old man underwent SEMS for obstructive rectal cancer. After successful placement of the SEMS, we performed a scheduled second total colonoscopy and detected 2 synchronous tumors: 1 in the ascending and 1 in the transverse colon $(\bullet$ Fig.3). For the ascending tumor, which was a $15-\mathrm{mm}$ flat elevated lesion (Type 0 -IIa), we performed EMR and pathologically found that the lesion type was tubular adenoma. For the transverse colon cancer, which was a $20-\mathrm{mm}$ flat elevated lesion (Type 0-IIa), we successfully performed EMR. The histologic findings revealed that the lesion type was intramucosal carcinoma (pTis) and the margins were free of carcinoma cells. Our results indicated the need to perform a low anterior resection (pT4, pN1, cM1).

\section{Discussion \\ $\nabla$}

In recent years, complete colonoscopy after SEMS placement has been recognized as feasible [5-7]. However, the feasibility of endoscopic resection for synchronous early colon cancer after SEMS placement is still unknown. To date, few reports have described the use of endoscopic resection for synchronous early colon cancer after SEMS placement $[5,7]$. Lim et al. reported a case 


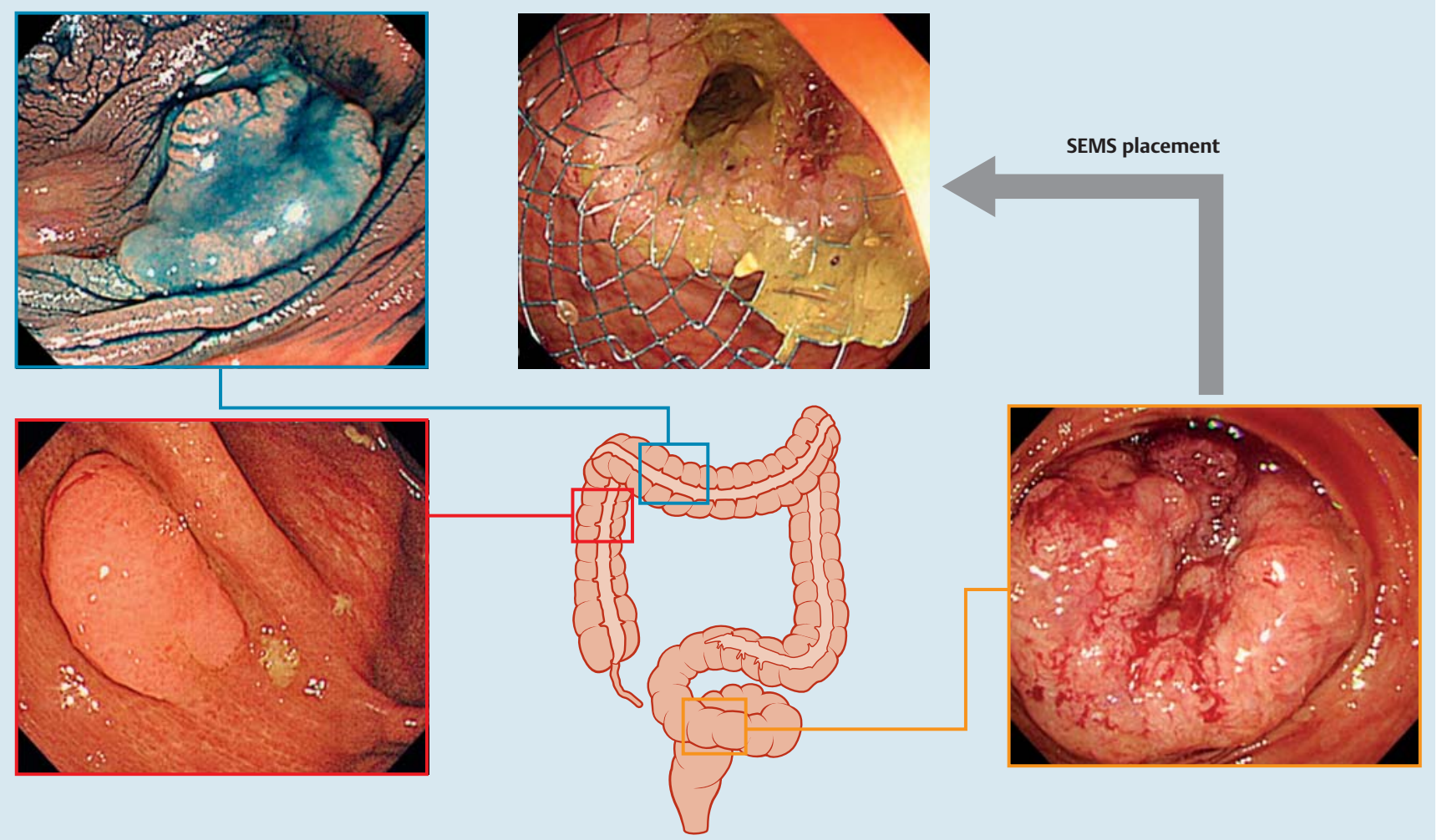

Fig. 2 SEMS placement in the ascending colon (orange square). We curatively resected the adenoma in the ascending colon (red square) and the early colon cancer in the transverse colon (blue square) with EMR.
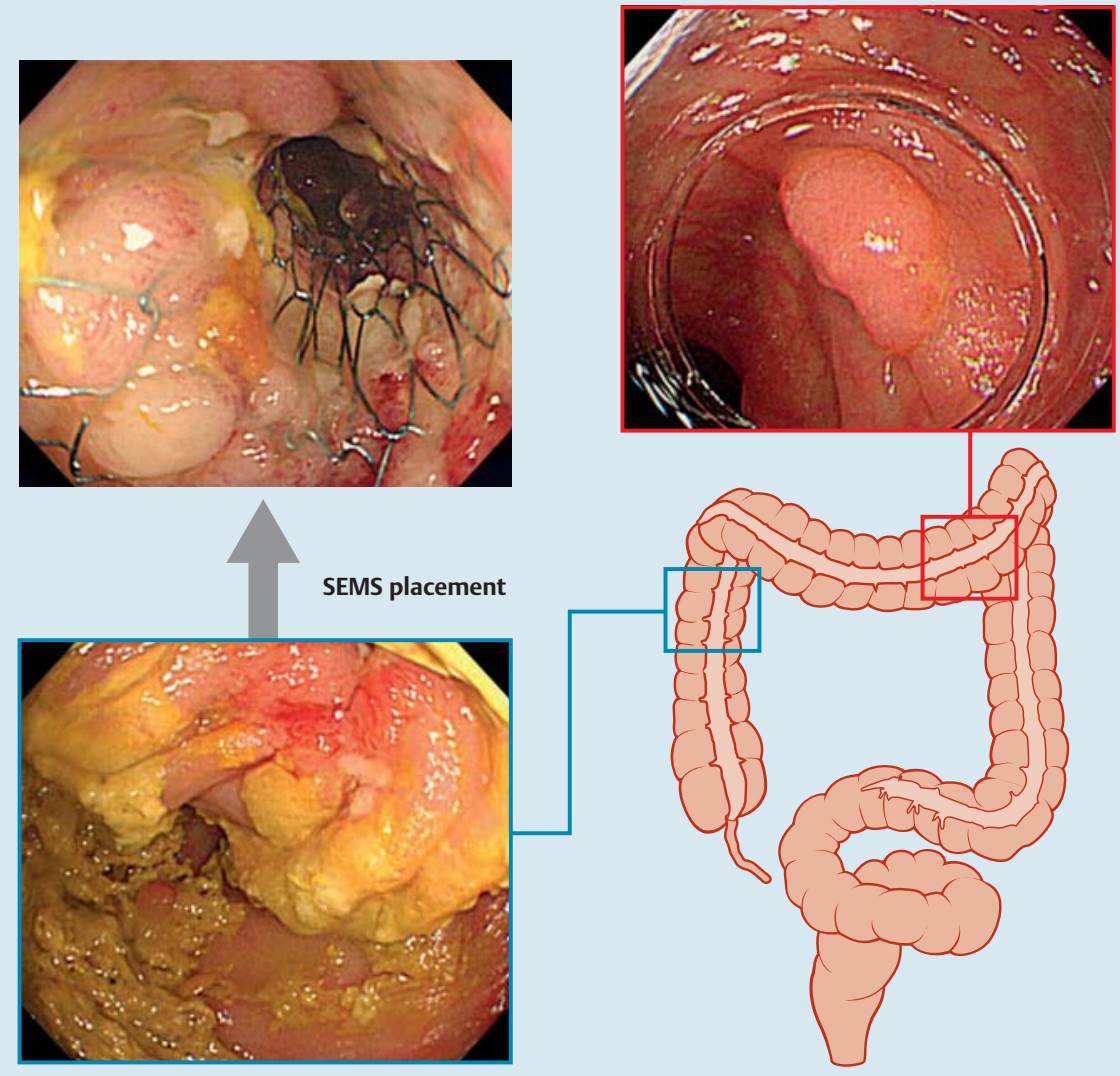

Fig. 3 SEMS placement in the ascending colon (blue square). We curatively treated the early colon cancer in the transverse colon with ESD (red square). 
involving early ascending colon cancer accompanied by obstructive sigmoid colon cancer [7]. These authors successfully treated the ascending colon cancer with EMR using a colonic stent. Similarly, Kim et al. reported a case involving early rectal cancer accompanied by obstructive advanced rectal cancer [5], and they treated the early one, which was located in the distal side of the SEMS, with ESD. The 3 cases reported herein are similar to those described previously; however, to the best of our knowledge, this is the first report to describe cases involving the treatment of synchronous early colon cancer with ESD using a colonic stent. The indication for SEMS placement is colonic obstruction due to a malignant colonic lesion. We selected Niti-S ${ }^{\circledR}(22 \mathrm{~mm}$ or 18 -mm diameter, 80 -mm length, TaeWoong Medical, South Korea) as our first line SEMS.

In our hospital, the average duration of SEMS placement before surgery was 56.4 days (data not shown). It has reported that surgery within 5 to 10 days is ideal for reducing stent-related complications. On the other hand, we also believe that it is important to improve the patient's general condition and examine the patient's disease precisely before surgery. In particular, in addition to cancer staging or checking cardiopulmonary functions, screening for synchronous colon cancers and endoscopic treatment of early synchronous cancers is essential before surgery. Therefore, we often needed more than 10 days before surgery. To date, we have not observed any complications such as re-obstruction, bleeding, or perforation before surgery.

For the second colonoscopy via SEMS, we selected a small-diameter colonoscope (e.g., PCF-PQ260 L, Olympus Medical, Tokyo, Japan). Polyethylene glycol was used for colonoscopy preparation for the second colonoscopy via SEMS. The average duration between second colonoscopy and SEMS placement was 14.9 days. We have performed colonoscopy through a SEMS for 12 patients so far and have detected synchronous cancers in 5 patients.

We select a small-diameter colonoscope without any magnifying function for the second colonoscopy. We diagnosed the lesions mainly with white light with indigo carmine dye spraying. Generally, it is possible to diagnose the characteristics of colonic neoplasms with white light endoscopy with indigo carmine dye with approximately $80 \%$ accuracy. Pit patterns or narrow band imaging diagnosis using magnifying endoscopy is useful and provides a much higher confidence in the diagnosis. These modalities are ideal to diagnose synchronous lesions by using magnifying endoscopy.

The indication for ESD was a large neoplasm that was suspected to be malignant. A small-diameter colonoscope was selected for endoscopic resection (e.g., PCF-Q260JI or PCF-Q260AZI, Olympus Medical, Tokyo, Japan).

Our results indicate that preoperative endoscopic treatment for synchronous early colon cancer after SEMS placement has a great advantage in that we can establish an appropriate surgical strategy for the patient. Endoscopic resection for synchronous early colon cancer enables identification of precise pathological information about a lesion compared with simple endoscopic observation. For cases in which lesions can be curatively treated with endoscopic resection, we can shorten the length of colectomy and accomplish a single-stage surgical operation. Even if a lesion cannot be resected using the endoscopic technique, we believe that surgical resection of the lesion is necessary and a multi-stage operation should be avoided.
Other modalities, such as computed tomography colonography [9] and magnetic resonance-colonography [10], have been reported to be effective for screening of the entire colon after SEMS placement. Although these minimally invasive modalities provide a high rate of discovery of synchronous lesions, scheduled second colonoscopies using stents have a great advantage in that they enable detection of lesions and biopsy or treatment of them with EMR and ESD.

The safety of total colonoscopy with SEMS has been reported in previous studies, which revelaed no major complications, such as massive bleeding or perforation [6,7]. In our cases and in previous cases [5,7], there were no complications associated with the endoscopic treatment. Because the number of cases is still inadequate, further investigations are needed to evaluate the safety of preoperative endoscopic treatment for synchronous early colorectal cancers after SEMS placement.

\section{Conclusions \\ $\nabla$}

In conclusion, preoperative endoscopic resection combined with the ESD technique for synchronous colorectal neoplasms after SEMS placement is thought to be effective as a surgical strategy for patients with malignant colorectal obstruction. However, evaluation of more cases is necessary to assess the safety of these techniques.

\section{Competing interests: None}

\section{References}

1 Khot UP, Lang AW, Murali K et al. Systematic review of the efficacy and safety of colorectal stents. Br J Surg 2002; 89: 1096-1102

2 Watt AM, Faragher IG, Griffin TT et al. Self-expanding metallic stents for relieving malignant colorectal obstruction: a systematic review. Ann Surg 2007; 246: 24-30

3 Moroi R, Endo K, Ichikawa $R$ et al. The Effectiveness of self-expandable metallic stent insertion in treating right-sided colonic obstruction: a comparison between SEMS and decompression tube placement and an investigation of the safety and difficulties of SEMS insertion in right colons. Gastroenterol Res Pract 2014; 2014: 372918

4 Bat L, Neumann G, Shemesh E. The association of synchronous neoplasms with occluding colorectal cancer. Dis Colon Rectum 1985; 28 : $149-151$

5 Kim JS, Lee KM, Kim SW et al. Preoperative colonoscopy through the colonic stent in patients with colorectal cancer obstruction. World J Gastroenterol : WJG 2014; 20: 10570-10576

6 Vitale MA, Villotti G, d'Alba L et al. Preoperative colonoscopy after selfexpandable metallic stent placement in patients with acute neoplastic colon obstruction. Gastrointest Endosc 2006; 63: 814-819

7 Lim SG, Lee KJ, Suh KW et al. Preoperative colonoscopy for detection of synchronous neoplasms after insertion of self-expandable metal stents in occlusive colorectal cancer: comparison of covered and uncovered stents. Gut liver 2013; 7: 311-316

8 [Anonymous] The Paris endoscopic classification of superficial neoplastic lesions: esophagus, stomach, and colon: November 30 to December 1, 2002. Gastrointest Endosc 2003; 58: 3-43

9 Singh K, Narula AK, Thukral CL et al. Role of CT Colonography in Colonic Lesions and Its Correlation with Conventional Colonoscopic Findings. J Clin Diagn Res : JCDR 2015; 9: Tc14-18

10 Achiam MP, Logager V, Lund Rasmussen V et al. Perioperative colonic evaluation in patients with rectal cancer; MR colonography versus standard care. Acad Radiol 2015; 22: 1522-1528 